\title{
AVALIAÇÃO DE PARÂMETROS PARA O MONITORAMENTO DA INTERFERÊNCIA DE PLANTAS DANINHAS NA CULTURA DE SOJA (Glycine max L.) ${ }^{1}$
}

\author{
C.A. SPADOTTO ${ }^{2}$, D.A.S. MARCONDES ${ }^{3}$, C.A.R. da SILVA ${ }^{4}$ e S. DAMASCENO
}

\begin{abstract}
RESUMO
O presente estudo visa fornecer subsídios para o processo de tomada de decisão de controle das plantas daninhas baseado em nível de dano na produtividade de culturas agrícolas. Com o objetivo de avaliar o aproveitamento de parâmetros das plantas daninhas e das plantas de soja no monitoramento da interferência das plantas daninhas, foi realizado, em Botucatu (SP), um experimento sob condições de campo, com delineamento em blocos casualizados. A cultura foi mantida na presença ou ausência de comunidade vegetal infestante por diferentes períodos. O monitoramento

da interferência das plantas daninhas na cultura da soja pode se dar através do acúmulo total de matéria seca da comunidade vegetal infestante e dos seguintes parâmetros de crescimento das plantas de soja: número de folhas trifolioladas, área da lâmina foliar, acúmulo de matéria seca de folhas e total. O acúmulo de matéria seca de cada espécie de planta daninha isoladamente, não foi parâmetro eficiente para determinar-se interferência (competição) na cultura da soja.

Palavras-chave: plantas daninhas, interferência, monitoramento, soja, nível de dano.
\end{abstract}

\section{SUMMARY}

\section{EVALUATING PARAMETERS FOR MONITORING WEED INTERFERENCE ON SOYBEAN (Glycine max L.)}

This research aims to subsidize decision making for weed control based on crop yield threshold. Field experiment was conducted in Botucatu (São Paulo, Brazil), with the objective of evaluating the utilization of soybean and weed parameters for monitoring weed interference on soybean yield. The experiment was delineated in a randomized block design. The crop was kept weedy or weed-free for different

\section{INTRODUÇ̃̃O}

As plantas daninhas presentes em culturas agrícolas têm causando prejuízos, porém o controle através do uso indiscriminado de herbicidas além de onerar o produtor, pode

1 Trabalho realizado com suporte financeiro da FAPESP

2 Eng $^{\circ}$ Agr $^{\circ}$, M.Sc., Pesquisador da EMBRAPA/CNPDA, Caixa Postal 69, CEP 13820-000, JaguariúnalSP

3 Eng $^{\circ} \mathrm{Agr}^{\circ}$, PhD., Professor Assistente da UNESP/FCA, Botucatu/SP

$4 \mathrm{Eng}^{\circ} \mathrm{Arg}^{\circ}$, pós- graduado na UNESP/FCA, Botucatu/SP

5 Graduando do curso de Agronomia de UNESP/FCA, BotucatwSP periods. TotaLweed dry matter and soybean parameters, such as number of leaves, leaf re, leaf dry matter and total dry matter, can be used for nitoring weed interference on soybean yield. Dry matter of each species separately, had no importance as a competitive element.

Keywords: Weeds, interference, monitoring, soybean, threshold.

trazer consequiências indesejáveis ao ambiente a à saúde humana.

Torna-se necessária a racionalização do uso de herbicidas. Mais que isto, é imprescindível a implementação do conceito de manejo da comunidade vegetal infestante em culturas agrícolas, conjugado com a integração de métodos de controle.

Para tanto, é de importância fundamental gerar informações visando o monitoramento da interferência de plantas daninhas em culturas agrícolas, e que permitam a determinação de níveis de dano no processo de tomada de decisão para a realização do controle. 
A preocupação conceitual com o manejo integrado de pragas na Entomologia Agrícola iniciou nos anos quarenta. No entanto, foi na década de 60 que este conceito começou a consolidar-se. Este conceito tem demonstrado ser de grande utilidade na racionalização do controle de insetos (10).

Nos últimos anos, tê m-se tentado introduzir os princípios e métodos do manejo integrado na área de estudo sobre plantas infestantes em culturas agrícolas. Entretanto, antes de adotar este conceito é necessário adaptá-lo.

A presença de plantas daninhas pode causar restrições de recursos, ou liberar para o meio substâncias aleloquímicas, interferindo no metabolismo da planta cultivada, causando efeitos negativos no crescimento, no desenvolvimento e, conseqüentemente, na produtividade da cultura agrícola.

O grau de interferência das plantas daninhas sobre culturas agrícolas é função de diversos fatores ligados à comunidade vegetal infestante, à cultura, ao ambiente, e ao período de convivência $(1,2,19)$. Devido ao grande número de fatores envolvidos, os resultados de pesquisa sobre a interferência de plantas daninhas em culturas agrícolas são bastante variados. Isto impossibilita a elaboração de recomendação generalizada do período adequado para realização do controle.

Trabalhos têm sido realizados com o objetivo de estudar a interferência de plantas daninhas em diferentes densidades $(12,13,20)$.

Diversos pesquisadores $(3,4,7,8,9,11,24)$ vêm se ocupando em estudos para o estabelecimento de níveis de danos na decisão do controle de plantas daninhas, baseados na densidade das diferentes espécies. Entretanto, os resultados estão sujeitos a uma série de variáveis, que se interrelacionam, o que dificulta a adoção de programas de controle baseados em densidades de espécies de plantas infestantes toleradas pela cultura.

Alguns autores estudaram as conseqüências da interferência de plantas daninhas sobre o crescimento de plantas de soja $(14,15,16,17,21,22,25,26)$.

O presente estudo visou fornecer subsídios para o processo de tomada de decisão de controle das plantas daninhas baseado em nível de dano na produtividade de culturas agrícolas.

\section{MATERIAL E MÉTODOS}

Experimento de campo foi conduzido em Botucatu (SP), no ano agrícola 1988/89.0 clima da região é temperado úmido, com verões quentes. $\mathrm{O}$ solo na área do experimento é Terra Roxa Estruturada distrófica, com textura muito argilosa, saturação em bases e acidez médias.

O preparo do solo foi convencional, com aração e gradagens, sendo a última gradagem realizada logo antes da semeadura. Com base nos resultados da análise química do solo, fez-se a calagem, e a adubação foi efetuada no sulco de semeadura.

A operação conjunta de adubação e semeadura foi mecanizada, resultando em uma densidade populacional de cerca de 20 plantas de soja por metro. Utilizou-se a cultivar IAC-12, de porte e ciclo médios, hábito de crescimento determinado, que floresceu aos 40 dias e completou o ciclo aos 125 dias.
As eventuais plantas gramíneas que emergiram na área do experimento, foram eliminadas em qualquer época do ciclo da cultura, através do uso de herbicida graminicida (sethoxydim) de aplicação pós-emergente. As principais espécies de plantas infestantes de folhas largas na área foram: mentrasto (Ageratum conyzoides L.), picão-preto (Bidens pilosa L.) e picão-branco (Galinsoga parviflora Cav.).

Os tratamentos foram divididos em dois grupos. Em um deles a cultura permaneceu na presença de comunidade infestante composta de plantas de folhas largas, desde a emergência, até as seguintes épocas do seu ciclo de desenvolvimento: $0,10,20,30,40,50$ e 60 dias. Nestas épocas foram feitas as coletas por amostragem aleatória na área útil das parcelas, após o que removeu-se as plantas infestantes através de capinas, mantendo-as sem infestação até a colheita da cultura.

No outro grupo a cultura permaneceu na ausência de comunidade vegetal infestante desde a emergência até diferentes épocas do seu ciclo: $0,10,20,30,40,50$ e 60 dias.

$\mathrm{O}$ experimento no campo obedeceu ao delineamento em blocos casualizados com quatro repetições. Cada parcela foi constituída de cinco linhas de semeadura, espaçadas de 0,6 m, com 5,0 m de comprimento. Como área útil de cada parcela consideraram-se as três linhas centrais, desprezandose $0,5 \mathrm{~m}$ em cada extremidade, totalizando 7,2 $\mathrm{m} 2$.

A coleta foi feita por amostragem aleatória emuma área total de $1,0 \mathrm{~m}^{2}$, composta por quatro subamostras de $0,25 \mathrm{~m} 2$ cada, dentro da área útil de cada parcela. As plantas infestantes foram identificadas, separadas por espécie e contadas. No laboratório, foram secas em estufas até peso constante. Então, foi determinado o acúmulo de matéria seca de cada espécie e o acúmulo total de matéria seca da comunidade vegetal infestante.

Também foram coletadas aleatoriamente dez plantas de soja por parcela ao término de cada período inicial, na presença e na ausência de comunidade vegetal infestante.

As plantas de soja foram levadas ao laboratório, onde avaliaram-se comprimento do caule, número de folhas trifoliadas e área da lâmina foliar em medidor de área. Depois disto, foram separadas em folhas e hastes (caule + pecíolos) para serem secas em estufa até peso constante, após o que, foi determinado o acúmulo de matéria seca de folhas e matéria seca total.

Com os dados obtidos calculou-se a diferença nos parâmetros estudados, entre as condições na presença e na ausência de plantas infestantes. Esta última foi considerada como padrão de crescimento da planta de soja.

As médias dos parâmetros amostrados independentemente a cada época, nas duas condições, foram comparados estatisticamente (23). Realizou-se, também, a análise de regressão entre a diferença de cada parâmetro e a produtividade da cultura.

Por ocasião da colheita da cultura, coletou-se manualmente o total de plantas de soja da área útil de cada parcela, que foram posteriormente trilhadas mecanicamente e pesada a produção de grãos.

Por se tratarem de tratamentos quantitativos (18), os dados de produtividade da cultura (em $\mathrm{kg} / \mathrm{ha}$ ) foram ajustados conforme adaptação do modelo de regressão "Broken-Stick" $(5,6)$. 
Os tratamentos em que a cultura foi mantida por variados períodos na presença de comunidade vegetal infestante, foram usados para determinar a extensão do período inicial de convivência possível, sem que a produtividade da cultura fosse reduzida, ou seja, serviram para determinar o início da interferência, baseando-se nos dados de produtividade da soja.

\section{RESULTADOS E DISCUSSÃO}

No Quadro 1 são apresentados os dados de produtividade média, variando com os diferentes períodos iniciais da cultura convivendo com a comunidade vegetal infestante.

Pelo ajuste dos dados conforme o modelo "BrokenStick" $(5,6)$ adaptado [Y= 2247,23 - 24,51 (x-49 + |x-49|],

\section{QUADRO 1 - Produtividade média da cultura de soja, variando com diferentes períodos iniciais de convivência com a comunidade vegetal infestante. Botucatu(SP), 1988/89.}

\begin{tabular}{c|c}
\hline $\begin{array}{c}\text { PERÍODO } \\
\text { CONVIVENCIA }\end{array}$ & $\begin{array}{c}\text { PRODUTIVIDADE } \\
\text { (kg/ha) }\end{array}$ \\
\hline- & 2355 \\
$0-10$ & 2313 \\
$0-20$ & 2140 \\
$0-30$ & 2297 \\
$0-40$ & 2131 \\
$0-50$ & 2221 \\
$0-60$ & 1732 \\
\hline F trat. & $1,2^{\bullet *}$ \\
C.V.(\%) & 17,9 \\
\hline
\end{tabular}

(1) dias após a emergència da cultura

* significativo a $1 \%$ de produtividade pode-se observar que a produtividade manteve-se estável até 49 dias do ciclo de cultura. Portanto, a interferência da comunidade vegetal infestante sobre a produtividade da cultura iniciou após 49 dias do ciclo da soja. Portanto, a interferência da comunidade vegetal infestante sobre a produtividade da cultura iniciou após 49 dias do ciclo da soja.

Os dados de acúmulo de matéria seca da comunidade vegetal infestante, levantados em diferentes épocas da cultura, aparecem no Quadro 2 e os de densidade no Quadro 3.

Pode-se observar que o acúmulo total de matéria seca da comunidade vegetal infestante manteve-se em níveis baixos até certo período inicial (0-20/30 dias), após o que ocorreu grande incremento; enquanto que com a densidade total, grandes incrementos já foram nos primeiros dias do ciclo da cultura.

Visando o aproveitamento dos dados de acúmulo de matéria seca da comunidade vegetal infestante no monitoramento da interferência das plantas daninhas, foi feita a análise de regressão deste parâmetro com a produtividade da soja.

A análise de regressão mostrou que a correlação linear entre o acúmulo total de matéria seca da comunidade vegetal infestante e a produtividade da cultura foi negativa e altamente significativa, segundo a equação:

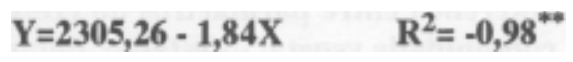

Entretanto, quando se considerou as espécies das plantas daninhas isoladamente, ou seja, cada uma das três espécies mais importantes (A. conyzoides, B. pilosa, G. parviflora), e as outras espécies reunidas em um só grupo, os coeficientes angulares (respectivamente 6,05, -13,13, 12,74 e -12,65) mostraram-se não significativos pelo teste " $t$ ".

Portanto, a importância de cada espécie isoladamente como elemento interferente (competitivo) foi pequena, devido provavelmente, às interações dentro da comunidade vegetal infestante, importando a resultante destas relações interativas para efeito de interferência à cultura.

As diferenças nos parâmetros do crescimento das plantas de soja, coletadas nas condições de presença e de ausência de plantas infestantes, em cada época pré-determinada, estão no Quadro 4.

QUADRO 2 - Acúmulo de matéria seca da comunidade vegetal infestante em diversas épocas da cultura de soja, variando com diferentes períodos iniciais de convivência. Botucatu(SP), 1988/89.

\begin{tabular}{c|c|c|c|c}
\hline \multirow{2}{*}{$\begin{array}{c}\text { PERÍODO }^{(\mathbf{1})} \text { DE } \\
\text { CONVIVÊNCIA }^{*}\end{array}$} & \multicolumn{4}{|c}{ ACÚMULO DE MATÉRIA SECA (g/m $^{\mathbf{2}}$} \\
\cline { 2 - 5 } & TOTAL & AGECO $^{(\mathbf{2})}$ & BIDPI $^{(\mathbf{3})}$ & GASPA $^{(\mathbf{4})}$ \\
\hline $0-10$ & 1,8 & 0,1 & 0,3 & 0,6 \\
$0-20$ & 5,8 & 0,5 & 0,3 & 2,2 \\
$0-30$ & 15,4 & 2,7 & 2,1 & 5,6 \\
$0-40$ & 40,6 & 7,8 & 7,1 & 12,8 \\
$0-50$ & 87,2 & 16,9 & 19,6 & 25,4 \\
$0-60$ & 94,1 & 18,4 & 13,7 & 10,1 \\
\hline
\end{tabular}

(1) dias após a emergência da cultura

(2) Ageratum conyzoides L

(3) Bidens pilosa L.

(4) Galinsoga parviflora L. 
QUADRO 3 - Densidade da comunidade vegetal infestante em diversas épocas da cultura de soja, variando com diferentes períodos iniciais de convivência. Botucatu(SP), 1988/89.

\begin{tabular}{c|c|c|c|c}
\hline \multirow{2}{*}{$\begin{array}{c}\text { PERÍODO } \\
\text { CONVIVÊNCIA }\end{array}$} & \multicolumn{4}{c}{ DENSIDADE (ind./m $\mathbf{m}^{\mathbf{2}}$} \\
\cline { 2 - 5 } & TOTAL & AGECO $^{(\mathbf{2})}$ & BIDPI $^{(\mathbf{3})}$ & GASPA $^{(\mathbf{4})}$ \\
\hline $0-10$ & 531 & 6 & 49 & 342 \\
$0-20$ & 915 & 119 & 42 & 605 \\
$0-30$ & 1104 & 429 & 70 & 450 \\
$0-40$ & 1172 & 563 & 72 & 374 \\
$0-50$ & 1186 & 655 & 56 & 323 \\
$0-60$ & 1479 & 859 & 111 & 226 \\
\hline
\end{tabular}

(1) dias após a emergência da cultura

(2) Ageratum conyzoides L

(3) Bidens pilosa L.

(4) Galinsoga parviflora L

QUADRO 4 - Diferença entre parâmetros do crescimento das plantas se soja na condiçáo de presença e ausência de comunidade vegetal infestante. Botucatu(SP), 1988/89.

\begin{tabular}{c|cccccc}
\hline PERÍODO $^{(\mathbf{1})}$ & $\mathbf{C C A}^{(\mathbf{2})}$ & $\mathbf{N F L}^{(\mathbf{3})}$ & $\mathbf{A L F}^{(\mathbf{4})}$ & $\mathbf{A M S F}^{(\mathbf{5})}$ & $\mathbf{A T M S}^{(\mathbf{6})}$ \\
\hline $0-30$ & $2,3^{\text {ns }}$ & $0,8^{*}$ & $88,0^{\text {ns }}$ & $0,2^{\text {ns }}$ & $0,3^{\text {ns }}$ \\
$0-40$ & $3,5^{\text {ns }}$ & $1,8^{*}$ & $130,0^{*}$ & $0,4^{*}$ & $0,7^{*}$ \\
$0-50$ & $4,8^{\text {ns }}$ & $9,4^{*}$ & $785,7^{*}$ & $1,8^{*}$ & $3,9^{*}$ \\
$0-60$ & $5,8^{\text {ns }}$ & $10,9^{*}$ & $1220,9^{*}$ & $3,2^{*}$ & $6,5^{*}$ \\
\hline
\end{tabular}

(1) dias após a emergência da cultura

(2) comprimento do caule $(\mathrm{cm})$

(3) número de folhas trifolioladas

(4) área da làmina foliar $\left(\mathrm{cm}^{2}\right)$

(5) acúmulo de matéria seca de folhas (g)

(6) acúmulo total de matéria seca (g)

ns năo significativo estatisticamente

- significativo a 5\% de probabilidade

As diferenças nos parâmetros nas duas condições foram constatadas somente após 20 dias do ciclo da cultura, e foi a partir de 40/50 dias que houve grande incremento destas diferenças, exceção feita à diferença no comprimento do caule.

Para o comprimento do caule não houve diferença estatisticamente significativa em todas as épocas. Para o número de folhas houve diferença significativa já aos 30 dias. A diferença para a área da lâmina foliar tornou-se significativa aos 40 dias. Quanto aos acúmulos de matéria seca de folhas e total, passou a haver significância aos 40 dias.

Foi feita a análise de regressão como conjunto de dados de diferença de cada parâmetro da planta cultivada com a produtividade, visando o aproveitamento no monitoramento da interferência das plantas daninhas. As correlações lineares são apresentadas no Quadro 5.

\section{CONCLUSÕES}

1. A interferência das plantas daninhas sobre a produtividade da cultura iniciou-se aos 49 dias do ciclo da soja.

2. O período inicial de convivência possível entre a soja e a comunidade vegetal infestante foi da emergência até 49 dias do ciclo da cultura.

4. O acúmulo total de matéria seca da comunidade vegetal infestante pode ser utilizado no monitoramento da interferência de plantas daninhas.

5. A importância de cada espécie infestante como elemento interferente (competitivo) isoladamente, foi pequena. 
QUADRO 5 - Coefíciente de correlação e equação de regressão do conjunto de dados da díferença de cada parâmetro da planta de soja com a produtividade da cultura. Botucatu(SP), 1988/89.

\begin{tabular}{|c|c|c|}
\hline PARÂMETRO & COEF. DE CORRELAÇÄO & EQUAÇĀO DE REGRESSĀO \\
\hline DIF. COMP. CAULE & $-0,82^{\text {ns }}$ & $\longrightarrow$ \\
\hline DIF. NÚM. FOLHAS & $-0,99^{* *}$ & $Y=2283,87-15,71 X$ \\
\hline DIF. ÁREA LÂM. FOLIAR & $-0,99^{* *}$ & $Y=2283,86-0,19 X$ \\
\hline DIF. ACÚM. MAT. SECA FOLHAS & $-0,99^{* \bullet}$ & $Y=2286,73-83,30 X$ \\
\hline DIF. ACÚM. TOTAL MAT. SECA & $-0,99^{* \bullet}$ & $Y=2282,86-37,64 X$ \\
\hline
\end{tabular}

* significativo a $1 \%$ de probabilidade

5. Parâmetros das plantas de soja, como número de folhas trifolioladas, área da lâmina foliar, acúmulo de matéria seca de folhas e total, podem ser usados no monitoramento da interferência das plantas daninhas na cultura.

\section{AGRADECIMENTOS}

Ao Prof. Dr. Robinson A. Pitelli (UNESP/FCAV, Jaboticabal/SP), ao Prof. Dr. Roberto A. Rodella (UNESP/Instituto de Biociências, Botucatu/SP), pelas importantes sugestões, e à Srta. Maria Amélia de Toledo Leme (EMBRAPA/CNPDA), pela revisão deste artigo técnicocientífico.

\section{LITERATURA CITADA}

1- BLANCO, H.G. A importância dos estudos ecológicos nos programas de controle de plantas daninhas. $O$ Biológico, São Paulo, 38(10):343-350, 1972.

2 - BLEASDALE, J.D.A. Studies on plant competition. In: HARPER, J.L. The biology of weeds. Oxford, Blackwell, 1960. p.133-142.

3 - CARLSON, H.L.; HILL, J.E. Wild oat (Avena fatua) competition with spring wheat: plant density effects. Weed Science, Champaign, 33:176-181, 1985.

4 - COBLE, H.D. Using economic thresholds for weeds in soybeans. In: CONFERENCIA MUNDIAL DE INVESTIGACIÓN EN SOJA, IV, 1989, Argentina. Anais, 1989, p. 1607-1612.

5 - COLWEEL, J.D. Fertilizer requirements. In: CSIRO. SOILS: an Australian viewpoint. Melbourne, 1983, p. 795-815.

6 - COLWELL, J.D.; SUHET, A.R.; VAN RAID, B. Statistical procedures for developing general soil fertility models for variable regions. Melbourne, CSIRO, Division of Soils, 1988. (Divisional Report, 93).

-7- COUSENS, R. A simple model relating yield loss to weed density. Annals of Applied Biology, Wellesbourne, 107:239-252, 1985.

8 - COUSENS, R. An empirical model relating crop yield to weed and crop density and a statistical comparison with other models. Journal of Agricultural Science, Cambridge, 105:513-521, 1985.
9 - DEW, D.A. An index of competition for estimating crop loss due to weed. Canadian Journal of Plant Science, Ottawa, 52:921-927, 1972.

10 - FERNÁNDEZ-QUINTANILLA, C.; ANDUJAR, J.L.G. Utilización del concepto de umbrales de decision para el control de malas hierbas. Información Técnica Económica Agraria, Madri, 75:57-69, 1988.

11- GEROWITT, B.; HEITEFUSS, R. Weed economic thresholds in cereals in Federal Republ ic of Germany. Crop Protection, Guildford, 9:323-331, 1990.

12 - HARRIS, T.C.; RITTER, R.L. Giant green foxtail (Setaria viridis var. major) and fall panicum (Panicum dichotomiflorum) competition in soybeans (Glycine max.). Weed Science, Champaign, 35:663-668, 1987.

13 - HENRY, W.T.; BAUMAN, T.T. Zone of competitive influence of two large seeded annual broadleaf weeds in soybeans. In: NORTH CENTRAL WEED CONTROL CONFERENCE, St. Louis, MO, XI II. 1985, Proceedings. Champaign: NCWCC Inc., 1985, p.6.

14 - HULBERT, J.C. Common cocklebur and tall morningglory emergence and interference in soybeans. Dissertation Abstracts International. Section B. Sciences and Engeneering, Ann Arbor, 46(2):355, 1985.

15 - LEGUIZAMON, E.S.; BODRER, M.L.; MACOR, L. Interferencia y control de ceboll in (Cyperus rotundus) en soja. Malezas, Buenos Aires, 11(4):209-216, 1985.

16 - MOSIER, D.G.;OLIVER, L.R. Common cocklebur (Xanthium pensylvanicum) and entire morningglory (Ipomoea hederacea var. integriuscula) interference in soybeans. In: SOUTHERN WEED SCIENCE SOCIETY ANNUAL MEETING, XXXVI, 1983, Biloxi, MS. Proceedings. Champaign: Southern Weed Science Society, 1983, p.81-82.

17 - MUNGER, P.H.; CHANDLER, J.M. Water relations and selected morphological measurements in soybeans-velvetleaf competition. In: SOUTHERN WEED SCIENCE SOCIETY ANNUAL MEETING, XXXVIII, 1985, Houston. Proceedings. Champaign: Southern Weed Science Society, 1985, p. 455.

Planta Daninha, v. 10, n. 1/2, 1992. 
18 - PIMENTEL GOMES, F. A estatística moderna na pesquisa agropecuária. 3. ed. Piracicaba: POTAFÓS, 1987, 160p.

19 - PITELLI, R.A. Interferência de plantas daninhas em culturas agrícolas. Informe Agropecuário, Belo Horizonte, 11(129):16-27, 1985.

20 - POHLAN, J. Results of interspecific competition between soybean (Glycine max) an Rottboellia exaltata. Bertrage Zur Tropischen Landwirtschaft and Veterinarmidizin, Berlin, 24(2):123-131. 1986.

21- ROSSI, C.A. Efeitos de períodos de controle e de convivência das plantas daninhas na cultura da soja (Glycine max). Jaboticabal: FCAV/UNESP, 1985. 49p. Trabalho de Graduação.

22 - SIKKEMA, P.H.; DEKKER, J. Use of infrared thermometry in determining critical stress periods induced by quackgrass (Agropyron repens) in soybeans (Glycine max). Weed Science, Champaign, 35:784-791, 1987.
23 - SNEDECOR, G.W.; COCHRAN, W.G. Statistical methods. 6.ed. Ames: The Iowa State University Press, 1978, 593p.

24 - THORNTON, P.K.; FAWCETT, R.H.; DENT, J.B.; PERKINS, T.J. Spatial weed distribution and economic thresholds for weed control. Crop Protection, Guildford, 9:337-342, 1990.

25 - VELINI, E.D. Avaliação dos efeitos de comunidades infestantes naturais controladas por diferentes períodos sobre o crescimento e produtividade da cultura de soja (Glycine max). Jaboticabal: FCAV/UNESP, 1989. 115p. Tese de Mestrado.

26 - WYSE, D.L.; YOUNG, F.L.; JONES, R.J. Influence of jerusalem artichoke (Helianthus tuberosus) density and duration of interference on soybean (Glycine max) growth and yield. Weed Science, Champaign, 34(2):243-247, 1986. 